\title{
Saxagliptin Anhydrous
}

National Cancer Institute

\section{Source}

National Cancer Institute. Saxagliptin Anhydrous. NCI Thesaurus. Code C75984.

The anhydrous form of saxag liptin, a potent, selective and competitive, cyanopyrrolidinebased, orally bioavailable inhibitor of dipeptidyl peptidase 4 (DPP-4), with hypoglycemic activity. Saxagliptin is metabolized into an, althoug h less potent, active mono-hydroxy metabolite. 